INGENIERÍA QUÍMICA

\title{
Biodiesel a partir de aceite de higuerilla utilizando lipasa inmovilizada
}

\author{
Alejandra Palomino*, Claudia C. Bocanegra*, Jorge E. López ${ }^{*}$, Luz M. Flórez** \\ Grupo Inter-institucional de Investigación en Biocombustibles, GRUBIOC \\ *Escuela de Ingeniería Química, Universidad del Valle. \\ **Dpto. Sistemas de Producción, Universidad Autónoma de Occidente \\ §e-mail: jelopez@univalle.edu.co
}

(Recibido: Junio 25 de 2009 - Aceptado: Junio 10 de 2010)

\section{Resumen}

Se analizó la transesterificación del aceite higuerilla con metanol, usando lipasa inmovilizada Mucor miehei como catalizador de la reacción y un aditivo solvente. Para evaluar el proceso y las condiciones de la reacción química, se aplicó un diseño de experimentos estadístico, variando las concentraciones del catalizador y del aditivo. Considerando que la viscosidad y el índice de acidez tienen un fuerte efecto sobre las características del biodiesel proveniente del aceite de higuerilla, estos parámetros se tomaron como variables de respuesta. Se encontró que las mejores condiciones de operación ocurren manteniendo una concentración en peso de enzimas cercanas al $13 \%$ y una concentración en volumen de aditivo promedio de 30\%. Con éstas condiciones, la viscosidad tiene valores cercanos a $59 \mathrm{~mm}^{2} / \mathrm{s}$ y el índice de acidez a $6 \mathrm{mg}$ de $\mathrm{NaOH}$ por gramo de muestra. En el rango de las condiciones encontradas más apropiadas, se caracterizaron los métil ésteres durante el transcurso de la reacción. La conversión más rápida de la reacción tuvo lugar en las primeras cuatro horas (más de $30 \%$ en peso de ésteres metílicos en los productos), luego ésta velocidad disminuye progresivamente hasta obtener valores de ésteres metílicos mayores al $45 \%$ alrededor de las siete horas.

Palabras Claves: Aceite de higuerilla, Biodiesel, Lipasa inmovilizada, Solvente.

CHEMICAL ENGINEERING

\section{Biofuel production from castor oil with immobilized lipase}

\begin{abstract}
Using as catalyst a immobilized lipase Muchor miehi and a solvent additive, castor oil enzymatic transesterification with methanol was analyzed. In order to evaluate the process and the chemical reaction conditions, a statistical experiment design was applied by varying the catalyst and additive concentrations. Since viscosity and acidity index have a strong effect on bio-diesel characteristics from castor oil, these parameters were taken as the comparative response variables. It was found that the best operation condition occurred when a weight concentration of enzymes was near $13 \%$ and additive volume concentration was near $30 \%$. With these conditions, the viscosity had values close to $59 \mathrm{~mm}^{2} / \mathrm{s}$ and the acidity had values of $6 \mathrm{mg} \mathrm{NaOH}$ by gram of sample. Within the range of the most appropriate conditions, methyl esters were characterized during the reaction. The fastest conversion reaction took place in the first four hours (more than $30 \% \mathrm{w} / \mathrm{w}$ of methyl esters in the products), then this velocity decreased progressively until it obtained a concentration of methyl esters higher than $45 \%$ at approximately seven hours.
\end{abstract}

Keywords: Castor oil, Bio-diesel, Immobilized lipase, Transesterification. 


\section{Introducción}

El gobierno Colombiano realizó la incursión en el mercado de biodiesel a partir del aceite de palma, pero es muy pertinente buscar otras alternativas de materias primas, debido a que un país no puede depender bioenergéticamente de un solo recurso. Además se deben buscar materias primas que no compitan con los alimentos ni por el uso de suelo, que tengan un ciclo productivo corto y que el desarrollo del cultivo mejore la calidad de los suelos. En ese sentido, la higuerilla es una muy buena opción (Lobato, 2007), aunque es un aceite muy fino, tiene una viscosidad alta y su productividad por hectárea no es tan buena como otras oleaginosas. Pero especialmente por la gran posibilidad de tener promisorios productos, en los últimos dos años se ha estado masificando progresivamente su cultivo, por lo tanto, se espera que esta oleaginosa pronto se convierta en una alternativa para producir productos especiales y biodiesel, con los excedentes. Otro estímulo que hay que tener en cuenta, es que para exportar y cumplir con las metas del gobierno colombiano, relacionadas con el aumento de la mezcla de biodiesel con diesel a un 20\% (B20) en el año 2012, se requieren más de 805,000 toneladas de biodiesel por año (Jaramillo, 2007) y no es suficiente la actual producción de palma africana y su proyección.

Un proceso innovador en el proceso de producción de biodiesel, es utilizar catálisis heterogénea, dado que así se puede recuperar el catalizador para reutilizarlo, evitando que éste contamine el producto y los efluentes. En Colombia hay muy pocos estudios de esta naturaleza, para el caso del proceso de biodiesel a partir de cultivos colombianos de higuerilla. Por esta razón, el objetivo principal del presente trabajo, fue evaluar las condiciones básicas más apropiadas para la transesterificación del aceite de higuerilla usando un biocatalizador heterogéneo.

Aunque la transesterificación con catalizadores homogéneos alcalinos tiene altos niveles de conversión, la reacción tiene algunas falencias: es intensiva en energía, es difícil la separación de glicerol y el catalizador alcalino tiene que recuperarse generalmente con lavado con agua, por lo que se le debe reprocesar. Para solventar en parte estos problemas, se ha propuesto reemplazar los catalizadores homogéneos por catalizadores heterogéneos como las enzimas inmovilizadas. Estos catalizadores, tipo lipasas inmovilizadas, al estar soportadas en resinas son más estables y pueden ser fácilmente reutilizables, además tienen un buen potencial para la producción de biodiesel porque trabajan en condiciones suaves de operación, no necesitan alcohol en exceso, a diferencia del uso de hidróxidos no forman jabón y no hay compuestos solubles que queden en el combustible o en la glicerina, incluyendo el agua, por lo que su purificación es más simple (Castor et al., 2007, Ma \& Hanna 1990, Meher et al., 2006). Por el contrario, el uso de lipasas libres es más costoso y presenta algunas dificultades técnicas como la contaminación del producto con actividad enzimática residual (Vyas et al., 2010).

El uso a escala industrial de los biocatalizadores en mención, recientemente era limitado por el alto costo de las enzimas inmovilizadas, no obstante la empresa Novo Nordisk lanzó varias lipasas inmovilizadas al mercado a precios competitivos. Para el presente trabajo se utilizó la lipasa Mucor miehei esteroespecífica 1, 3 en su forma inmovilizada, debido a que en estudios previos mostró mayor conversión a métil ésteres (Lloyd et al., 1996, Castor et al., 2007).

\section{Metodología}

\subsection{Reactivos y materiales}

En la reacción se usó como biocatalizador, la lipasa inmovilizada estereoespecífica Mucor miehei (Lipozyme ${ }^{\circledR} \mathrm{IM}$ ), a la cual se le agregó hexano comercial como aditivo, para evitar su aglomeración. La transesterificación del aceite de higuerilla comercial, se hizo con metanol grado reactivo. El aceite higuerilla tuvo una viscosidad inicial de $195 \mathrm{~mm}^{2} / \mathrm{s} \mathrm{a} 40^{\circ} \mathrm{C}$.

\subsection{Métodos de análisis}

La concentración de metil ésteres en las muestras, se determinó en un HPLC marca Shimadzu modelo Prominance, utilizando como patrones los ácidos grasos ricinoléico, linolénico y linoléico, 
los cuales se derivatizaron en trifluoruro de boro con metanol $(14 \% \mathrm{v} / \mathrm{v})$. En este equipo se usó un detector de índice de refracción RID-10A, a una longitud de onda de $205 \mathrm{~nm}$. La fase móvil consistió en una mezcla de acetonitrilo $90 \%(\mathrm{v} / \mathrm{v})$ y agua y la separación cromatográfica se efectuó a un flujo de $1 \mathrm{ml} / \mathrm{min}$ a $50^{\circ} \mathrm{C}$, en una columna de partículas de $4 \mu \mathrm{m}$ C18 de dimensión $3.9 \mathrm{~mm}$ x 300 mm. La adquisición e integración de datos se realizó con el software LC Solution Analysis. Las muestras antes de analizarlas se ultracentrifugaron y se filtraron a través de un filtro de $0.45 \mu \mathrm{m}$.

A los productos de reacción del diseño experimental, se les midió la viscosidad con un viscosímetro Ostwald a $40^{\circ} \mathrm{C}$ (ASTM D 445) y se les determinó el índice de acidez con la norma técnica colombiana NTC 218.

\subsection{Procedimiento experimental}

La reacción de transesterificación se llevó a cabo en un vaso de precipitación en donde el aceite de higuerilla, el hexano y el metanol, se calentaron en un baño-maría, a la temperatura de reacción. La mezcla se dejó durante 30 minutos para garantizar el equilibrio. Se adicionó la enzima y al mismo tiempo se inició la agitación. Finalizado el tiempo de reacción, se detuvo la agitación y la mezcla inmediatamente se filtró. La fase liquida se llevó a un proceso de rota-evaporación a una presión de 300 mbar y a una temperatura de $40^{\circ} \mathrm{C}$. A estas condiciones se buscó que el solvente y el alcohol se evaporaran y que solo quedara una fase con aceite, glicerina y biodiesel para el análisis de viscosidad e índice de acidez.

\subsection{Diseño de experimentos}

Para el diseño experimental de la reacción de transesterificación, se siguió el método rotacional central compuesto. Según el análisis de la bibliografía consultada, fueron varias las variables que se dejaron fijas: la temperatura, relación molar metanol: aceite, la velocidad de agitación y el tiempo. Con respecto a la temperatura de reacción, ésta se estableció en $40^{\circ} \mathrm{C}$, porque es donde las lipasas como la Mucor miehei, la Candida antarctica y la Pseudomonas cepacia, en cualquier tipo de aceite presentan su mayor actividad y a una temperatura mayor empiezan a desnaturalizarse (Fukuda et al., 2001, Nie et al., 2006, Noureddini et al., 2005). La relación molar metanol:aceite, se fijó en 3:1 debido a que un mayor exceso de alcohol provoca la deshidratación de la enzima, como lo muestran los estudios de De Oliveira, et al., (2004) con etanol y aceite de higuerilla, y Castellanos \& Gonzáles (2004) y Nie, et al., (2006) quienes trabajaron con metanol y aceite de palmiste y oliva, respectivamente. La agitación se estableció en 200 rpm, ya que es suficiente para eliminar los problemas difusivos frente a la reacción y no tiene una velocidad muy grande como para fracturar la enzima, lo cual fue probado por De Oliveira et al., (2004) para aceite de higuerilla y por Lloyd et al., (1996) para aceite de soya y oliva, y sebo animal. El tiempo de reacción se estableció en un principio de 4 horas, considerando que ese tiempo era suficiente para detectar una cantidad apreciable de triglicéridos en los productos.

Por ser los costos un factor clave para la implementación del proceso a escala industrial, se consideraron la concentración del catalizador y la concentración del aditivo, como variables independientes del diseño de experimentos. Esta última se tomó en cuenta porque es necesario el uso de solventes no polares ( $\log \mathrm{P}>2$ ), para mejorar la solubilidad entre el triglicérido y el alcohol y mantener la actividad catalítica de la enzima (Nie et al., 2006, De Oliveira et al., 2004, Li et al., 2006). Además, no hay suficientes antecedentes sobre esta variable y durante las pruebas preliminares de exploración y familiarización se encontró, que un exceso del solvente influye fuertemente aglomerando la enzima, disminuyendo el área catalítica, ocasionando problemas operativos y difusionales. Se utilizó el n-hexano como aditivo, considerando el coste de producción industrial y debido a que la enzima Mucor miehei da mayores conversiones en presencia de este solvente (Delimitsou et al., 2002).

Los niveles de cada ensayo, se seleccionaron según los resultados de De Oliveira et al., (2004), y Lloyd et al., (1996), dejando los niveles de la concentración de solvente en un rango de 5 a $30 \%$ $(\mathrm{v} / \mathrm{v})$ con base en el volumen del aceite y el alcohol. Para la concentración de la enzima se 
siguió a Castellanos \& González (2004) y a Mittelbach (1990), manteniendo los valores en un rango de 3 a $13 \%(\mathrm{p} / \mathrm{p})$ con base en el peso del aceite.

Para la realización del modelo de optimización, se escogió un diseño rotacional central compuesto CCD ortogonal de dos factores, con dos variables de respuesta. Con ayuda del software STATGRAPHIS Plus versión 5.0, se realizó el análisis factorial para medir la influencia de las variables sobre la viscosidad y el índice de acidez. Luego por un desarrollo de regresiones y superficies de respuesta, se trató de optimizar simultáneamente la viscosidad y el índice de acidez, hacia los valores mínimos.

A las mejores condiciones encontradas anteriormente, se realizó un seguimiento cinético a la reacción, haciéndole algunas caracterizaciones a muestras periódicas que se tomaron durante la reacción.

\section{Resultados y Discusión}

\subsection{Evaluación de las condiciones de reacción}

Dentro de los rangos ensayados para las dos variables controlables, con un nivel de confianza del $95 \%$, se encontró que la concentración de la enzima es la que tiene un verdadero efecto sobre la viscosidad final de la mezcla del producto (ver Figuras 1 y 2), mientras que para la acidez las dos variables independientes son significativas, a pesar que la interacción entre ellas es despreciable (ver Figuras 3 y 4 ).

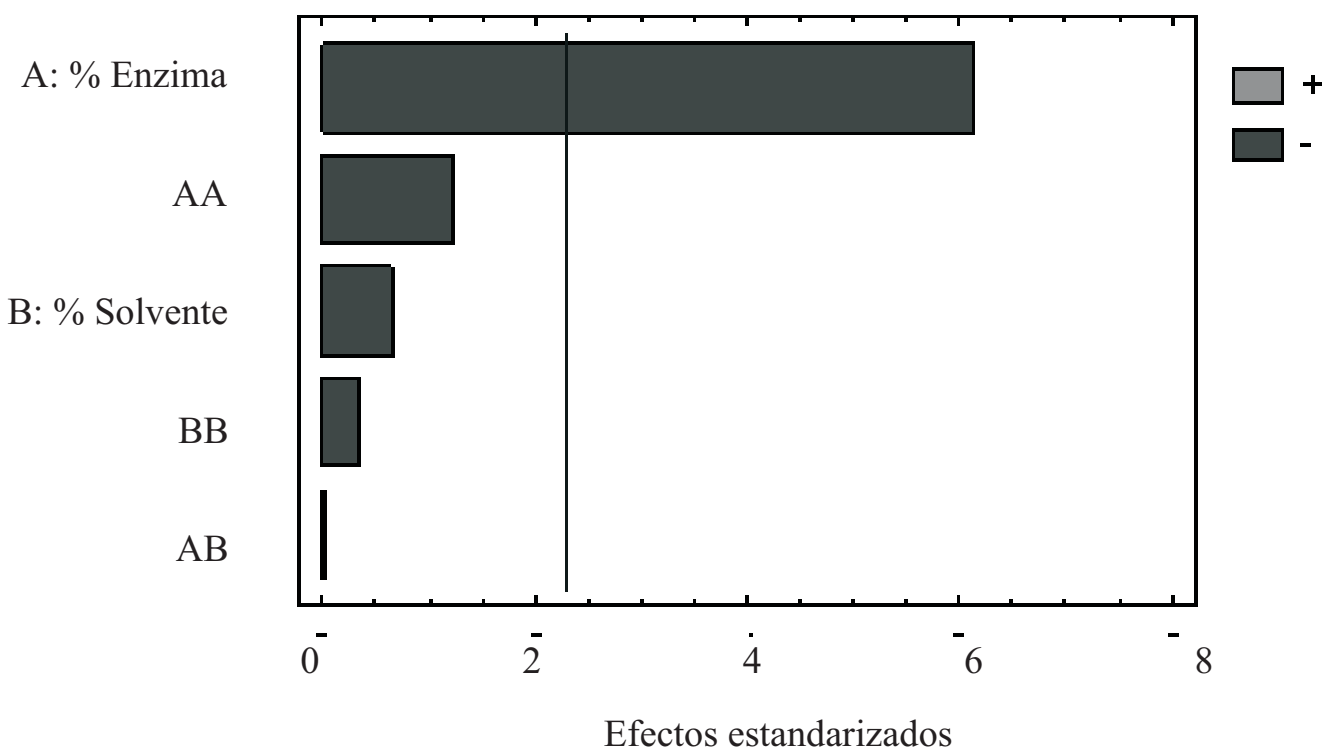

Figura 1. Diagrama de Pareto para la viscosidad (Variables normalizadas) 


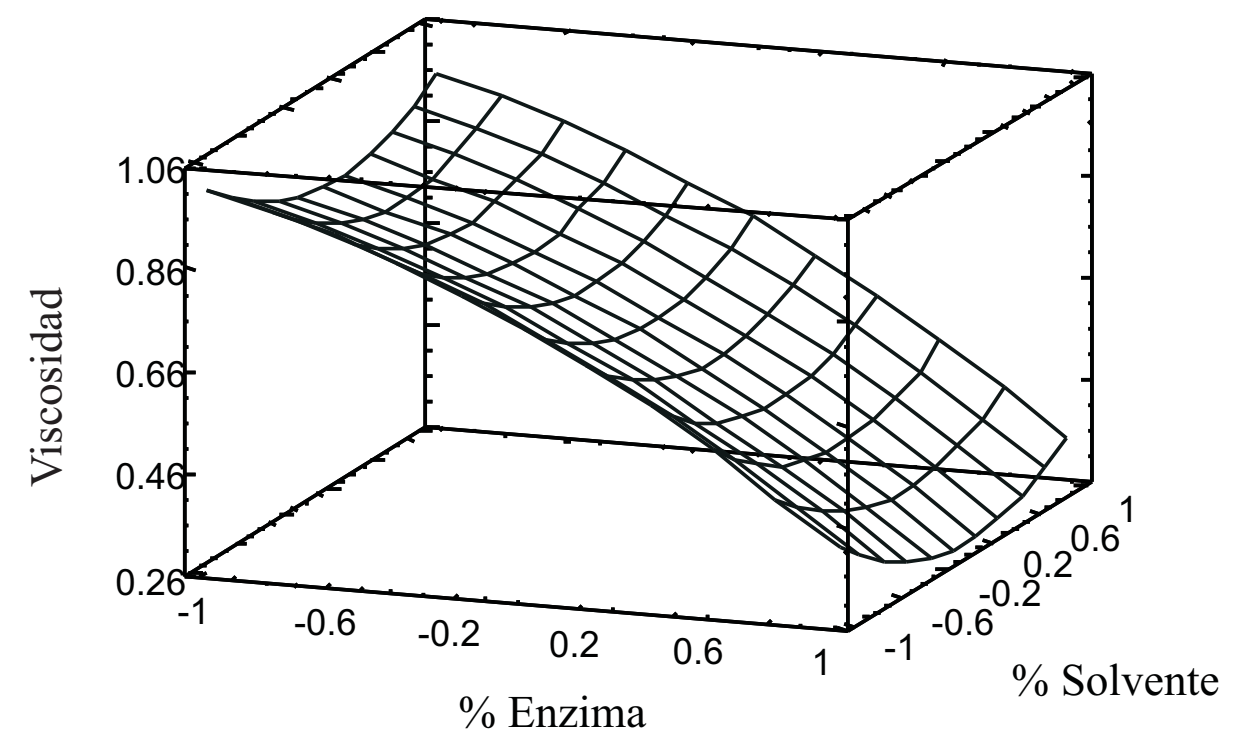

Figura 2. Superficie de respuesta para la viscosidad (Variables normalizadas)

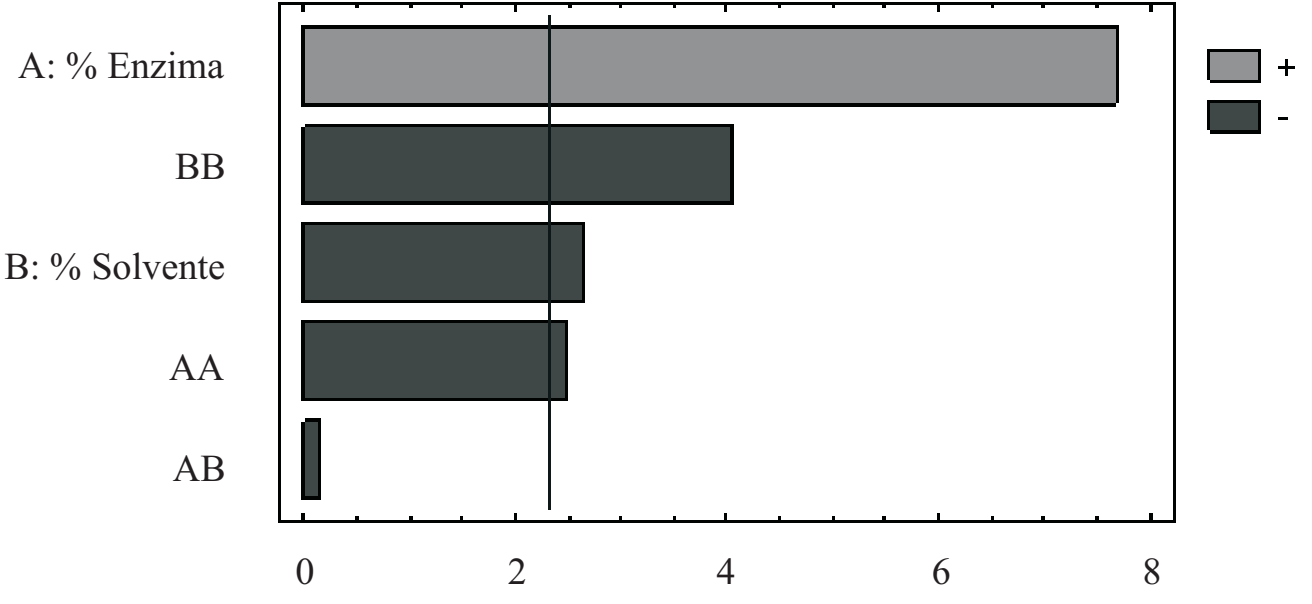

Efectos estandarizados

Figura 3. Diagrama de Pareto para el índice de acidez (Variables normalizadas) 


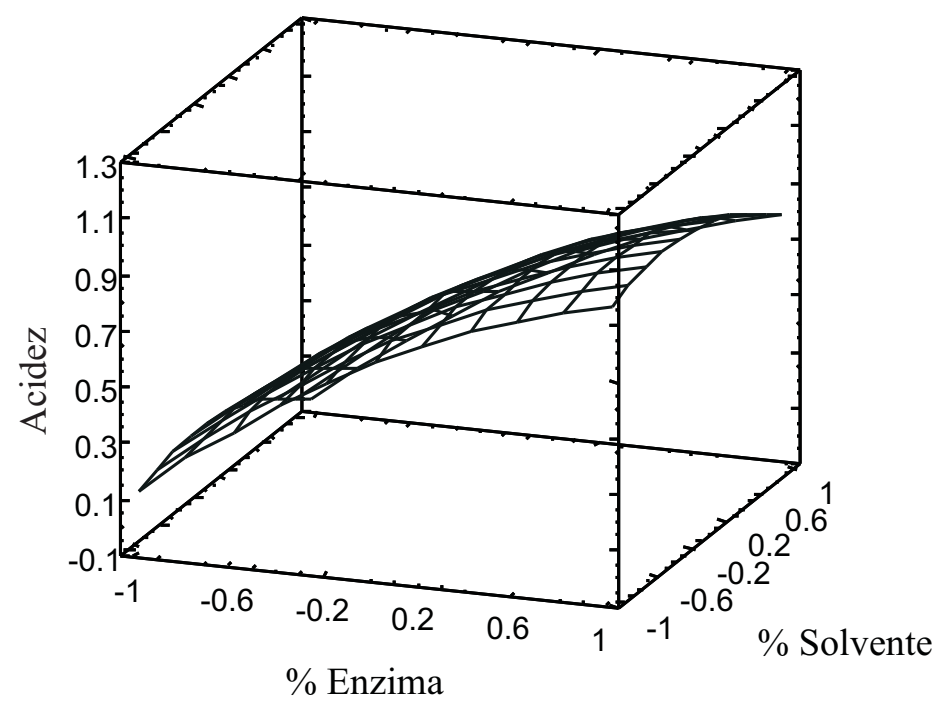

Figura 4. Superficie de respuesta para el índice de acidez (Variables normalizadas)

La concentración de la enzima aumenta la acidez, debido a que la enzima favorece tanto la reacción de hidrólisis como la transestrificación, aumentando la producción de ácidos grasos (Anthonsen \& Sjursens 2000, Kaieda et al., 2001, Shah et al., 2004). Se consideró que este efecto se debió a que la lipasa busca retener agua para mantener su flexibilidad y estructura o cambiándola ligeramente. Pero este estado de hidratación afecta apreciablemente la catálisis, ya que su actividad compite con el de mantener la adsorción del agua y/o producir hidrólisis, obstaculizando además los contactos directos entre las fases por la interposición quizás, de un mayor espesor de las películas de transferencia de masa o de sitios activos. Esta situación se dió, ya que la enzima permanecía expuesta al ambiente, mientras se estabilizaba la temperatura de reacción. Por el contrario, el aumento de la concentración de n-hexano disminuyó el índice de acidez, con lo cual podría comprobarse que este compuesto permite que la enzima mejore los problemas de tensión interfacial entre sus partículas sólidas y las moléculas de los productos. De esta manera se evita que los ácidos grasos, la glicerina y el biodiesel afecten el área expuesta del catalizador, haciendo que catalice más la reacción (Nie et al., 2006), que es dependiente de la cantidad de producto formado por área fija interfacial de la enzima, bajo las condiciones de operación (Puthli et al., 2006).

Dándole una mayor importancia a la viscosidad que al índice de acidez y de acuerdo con los resultados encontrados en la gráfica de superficie de respuesta (ver Figura 5), se consideró mantener una concentración de enzima de $13 \%$ y una concentración de solvente de $30 \%$, para tener las mejores condiciones del proceso. Con los demás parámetros fijos, a estas condiciones se obtuvo una viscosidad de $87.4 \mathrm{~mm}^{2} / \mathrm{s}$ y un índice de acidez de $23.8 \mathrm{mg} \mathrm{NaOH} / \mathrm{g}$ de biodiesel, en la mezcla final de la reacción, sin alcohol, lipasas y hexano en ese producto. Es posible que en la mezcla final quedaron no solo los metilésteres y los ácidos grasos del aceite de higuerilla que no reaccionaron con la glicerina, sino también diglicéridos y monoglicéridos; por lo tanto, el valor de la viscosidad y un poco menos el de la acidez del biodiesel puro, deben ser más bajos. 


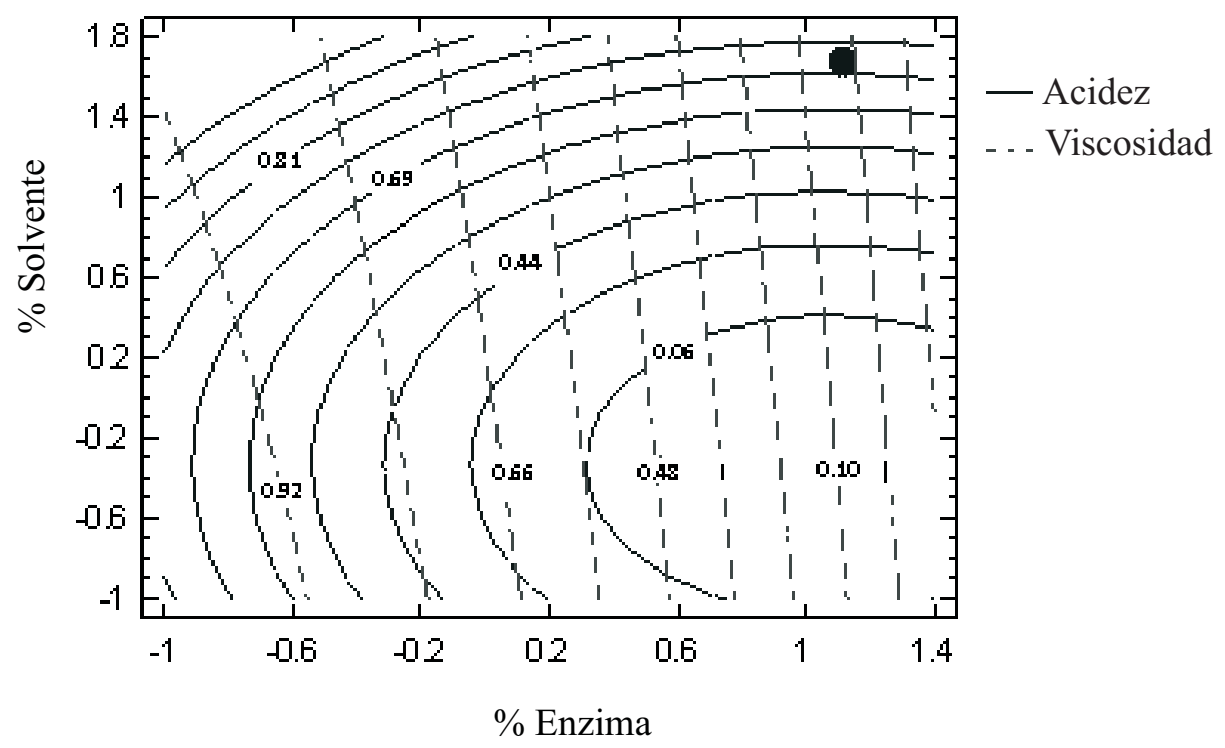

Figura 5. Superficie de contornos para la optimización simultánea (Variables normalizadas)

Tratando de evitar las interacciones de la lipasa con el medio ambiente, para evitar el problema de la hidratación de la enzima, ésta se pesó y adicionó cuando ya se tenía a la temperatura deseada, la mezcla de aceite de higuerilla, metanol y hexano. De esta forma se logró que la viscosidad final disminuyera en un $25 \%$ y el índice de acidez en un $79 \%$, incluso bajando la concentración del aditivo al 5\%. De esta forma, se corroboró la hipótesis de que la lipasa se hidrata muy fácilmente con el medio ambiente y por esto disminuye su actividad catalítica, además el requerimiento del aditivo para evitar la acción del agua, disminuye significativamente.

De acuerdo con la composición de los ácidos grasos presentes en el aceite de higuerilla, en el cual los ácidos ricinoleico, linoleico y linolénico son el 97\% de su composición, (Ogunniyi 2006), con las condiciones finalmente encontradas, a la reacción se le realizó un seguimiento a través del tiempo, determinando los metil ésteres derivados de esos ácidos grasos.

Estudios realizados por De Oliveira et al. (2004), se comenta que cuando la reacción se lleva a cabo en un medio acuoso, las conversiones que se alcanzan no son superiores al $40 \%$ de ésteres etílicos, muy similar a lo que se observó en la experimentación con una conversión a las 4 horas de la trasesterificación de $32.99 \%$ en peso. Sin embargo, se observó un aumento hasta un 40.97\% a las 8 horas (ver Figura 6). Una conversión más alta se alcanzó a las 7 horas con $46.28 \%$, en condiciones suaves de operación, $40^{\circ} \mathrm{C}, 200 \mathrm{rpm}$ y relación molar metanol:aceite de 3:1.

Al comparar los resultados obtenidos con los reportados en la literatura, y si se tiene en cuenta condiciones similares de operación, los mayores valores reportados son los obtenidos por Lloyd et al., (1996) que alcanzaron con la Mucor miehei conversiones hasta de 94.8 y $75.4 \%$ de ésteres metílicos con sebo y aceite de soya respectivamente a las 8 horas de reacción, utilizando el mismo alcohol y aditivo. Castellano \& González (2004) lograron rendimientos hasta del $71 \%$ de ésteres etílicos con la Lipozyme IM, sin el uso de aditivo, a partir de aceite de palmiste. Estos datos confirman que la conversión depende de la materia prima y del uso del aditivo, pero si éste último no se utiliza, hay muchos problemas operativos debido a la gran aglomeración de las partículas de lipasa. 


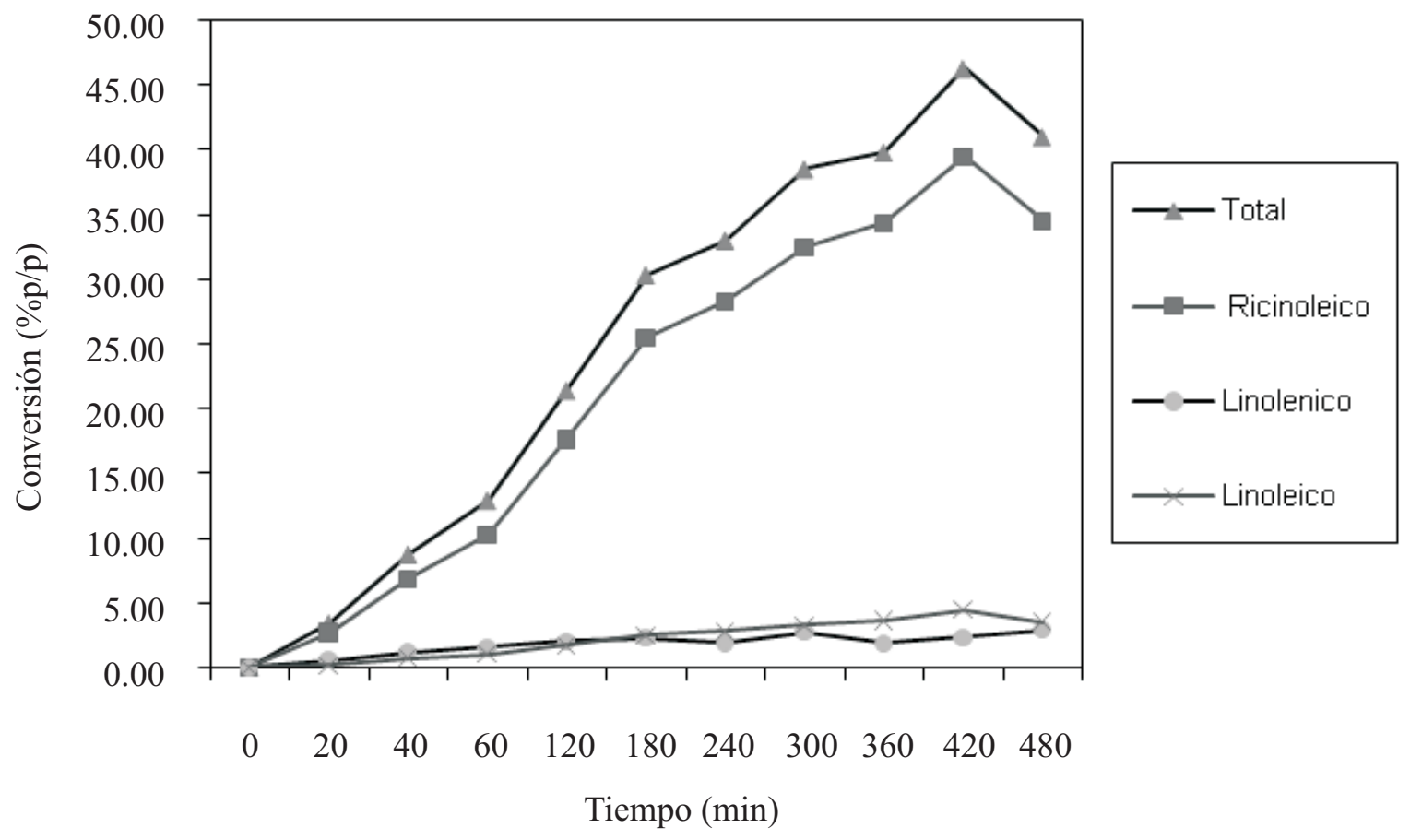

Figura 6. Variación de la conversión respecto al tiempo.

Aunque los rendimientos de la reacción estudiada no son tan altos como los obtenidos para otras materias primas, las suaves condiciones de operación encontradas, es un gran atractivo de la naturaleza de este primer estudio con aceite de higuerilla. Un mejor cuidado de la enzima utilizada para que no se hidrate y una optimización de las variables que se mantuvieron fijas, además de otros parámetros controlables, como la secuencia del mezclado y el tipo de agitación, seguramente mejorará la producción de metilésteres.

Por lo observado en este estudio, las hipótesis de los mecanismos enzimáticos que se proponen, son:

- Una etapa pre-estacionaria, en la que la concentración del complejo enzima-sustrato (ES) se da muy rápido, quizás del orden de segundos. Aquí la lipasa debe primero enlazarse con la interfase oleo-acuosa a través de un cambio conformacional antes de unirse al sustrato. Físicamente este envuelve un movimiento del "lid" y la enzima asume una conformación abierta (Whitaker, 2003).
- Una etapa de progreso continuo que se da inmediatamente después de la etapa preestacionaria hasta la hora 7 , donde lo que se forma de ES es igual a lo que reacciona en productos, más la enzima.

- Una etapa de bloqueo de la reacción o de los productos, a partir del cual hay reacciones reversibles o de degradación de los metil ésteres.

Debido al aumento del índice de acidez en el producto final, junto con la reacción principal puede ocurrir una reacción paralela de hidrólisis, esto a nivel de cinética enzimática se conoce como inhibición reversible incompetitiva de varios pasos (Mathews et al., 2005), el esquema de la reacción se muestra en la Figura 7.

La enzima (E) que se une al triglicérido (S1) forma la glicerina (P1) y un grupo acilo (A) que se prepara para recibir el metanol (S2) y formar el éster metílico (P2) pero el agua (I) en exceso compite en este paso, liberando los ácidos grasos (P3). 


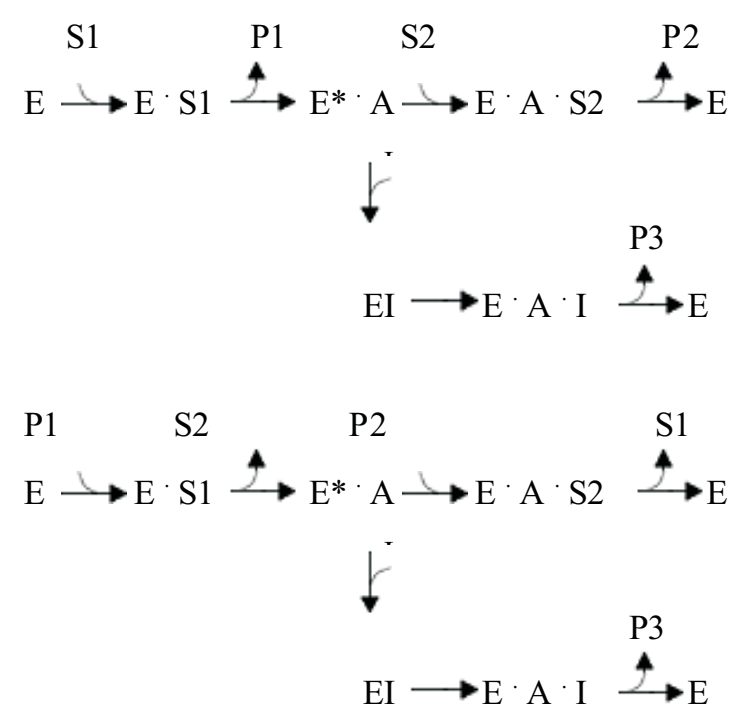

Figura 7. Mecanismo de reacción con inhibición competitiva.

\section{Conclusiones}

Empleando catálisis enzimática con el uso de Mucor miehei (Lipozyme ${ }^{\circledR}$ IM) es posible la obtención de biodiesel a partir del aceite de higuerilla utilizando metanol y solvente, con mínima producción de desechos contaminantes. Durante la experimentación no se presentó en ninguno de los ensayos formación de emulsiones $\mathrm{u}$ otro tipo de fenómenos que pudieran dificultar la purificación de biodiesel.

No es necesario el lavado ni el posterior secado del producto de la reacción para la purificación del biodiesel ni de la glicerina, debido a que el catalizador es retirado por filtración. El evitar la etapa de secado es un ahorro sustancial de energía, especialmente para el biodiesel de higuerilla debido a que el grupo OH- que esta presenta en su cadena, forma con el agua puentes de hidrogeno.

\section{Agradecimientos}

Los autores presentan sus agradecimientos al biólogo José L. López por la asistencia dada en los ensayos y a las entidades, que permitieron sus espacios, infraestructura y tiempos. a los investigadores del Grupo Interinstitucional de Biocombustibles GRUBIOC.

\section{Referencias bibliográficas}

Anthonsen, T., \& Sjursens, BJ. (2000). Importance of water activity for enzymes catalysis in nonaqueous organic systems. Methods in non-aqueous enzymology. New Dali, India: Ed. M.N, Gupta.

Castellano, M., \& Gonzales, A. (2004). Sintesis de ésteres alquílicos mediante alcoholisis del aceite del palmaste utilizando como catalizador lipasa de Mucor miehei. Trabajo de grado. Universidad Industrial de Santander. Escuela de Química. Colombia.

Castor, P., Coello, J., \& Castillo, L. (2007). Opciones para la producción y uso del biodiesel en el Peru. Lima, Peru: Soluciones Practicas Itdg.

Delimitsou, C., Zoumpanioti, M., Xenakis, A., \& Stamatis, H. (2002). Activity and stability studies of mucor miehei lipase immobilized in novel microemulsion-based organogels. Biocatalysis and Biotransformation. 20 (5), 319-327

De Oliveira, D., Luccio, M., Faccio, C., Dalla Rosa, C., Bender, J.P., N. Lipke, N., Menoncin, S., Amroginski, C., \& Oliveira, V. (2004). Optimization of enzymatic production of biodiesel from castor oil in organic solvent medium. Applied Biochemistry and Biotechnology 115 (1/3), 771-780.

Fukuda, H., Kondo, A., \& Noda, H. (2001). Biodiesel fuel production by transesterification of Oils. Journal of bioscience and bioengineering. 92 (5), 405-416.

Jaramillo, J. (2007) Seminario Presente y futuro de biocombustibles de Colombia. Federación Nacional de Biocombustibles de Colombia. Universidad Nacional de Colombia, sede de Palmira. Mar. 21

Kaieda, M., Samukawa, T., Kondo, A., \& Fukida, H. (2001). Effect of methanol and water contents on production of biodiesel fuel from plant oil catalyzed by various lipases in a solvent-free system. Journal of bioscience and bioengineering $91(1), 12-15$. 
Li, L., Du, W., Liu, D., Wang, L., \& Li, Z. (2006). Lipase-catalyzed transesterification of rapeseed oils for biodiesel production with a novel organic solvent as the reaction médium. Journal of Molecular Catalysis B: Enzymatic 43 (1-4), 58-62.

Lloyd, N., Foglia, T., \& Marmer, W. (1996). Lipase-Catalyzed Production of Biodiesel. Journal of the American Oil Chemists' Society 73 (9), 1191-1195.

Lobato V. (2007). Metodología para Optimizar el Análisis de Materias Primas para Combustibles en los Países del Cono Sur. Montevideo, Uruguay: PROCISUR IICA.

Ma, F., \& Hanna, M. (1999). Biodiesel Production: A Review. Bioresource Technology 70 (1), 1-15.

Mathews, C., Van Holde K., \& Ahern, K. (2005). Bioquímica. Ed. Pearson.

Meher, L.C., Vidya, D., \& Naik, S.N. (2006). Technical aspects of biodiesel production by Transesterification. Renewable \& Sustainable Energy Review. 10 (3), 248-268.

Mittelbach, M. (1990). Lipase catalyzed alcoholysis of sunflower. Journal of the American Oil Chemists' Society. 67 (3), $168-170$.
Nie, K., Xie, F., Wang, F., \& Tan, T. (2006). Lipase catalyzed methanolysis to produce biodiesel: Optimization of the biodiesel production. Journal of Molecular Catalysis B: Enzymatic 43, 142 - 147.

Nouredinni, H., Gao, S., \& Philkana, R.S. (2005). Immobilized pseudomonas cepacia lipase for biodiesel fuel production from soybean oil. Bioresource Technology. 96 (7), 769-777.

Ogunniyi, D.S. (2006). Castor Oil: a vital industrial raw material. Bioresource Technology. 97(9), 1086-1091.

Puthli, S., Rathod, K., \& Pandit, A. (2006). Enzymatic hydrolysis of castor oil: Process intensification studies. Biochemical Engineering Journal.31(1), 31-41.

Shah, S., Sharma, S., \& Gupta, M.N. (2004). Biodiesel preparation by lipase-catalyzed transeterification of jatropha oil. Energy \& Fuels 18 (1), 154-159.

Vyas A.P., Jaswant L., Verma, J.L., Subrahmanyam, N. (2010). A review on FAME production processes. Fuel 89 (1), 1-9.

Whitaker, J. R., Voragen, G. J., \& Wong, W.S. (2003). Handbook of food enzymology. New York, Estados Unidos. 